\title{
Enhanced Fuzzy Systems for Type 2 Fuzzy and their Application in Dynamic System Identification
}

\author{
Shun-Feng Su and Ming-Chang Chen \\ Department of Electrical Engineering \\ National Taiwan University of Science and Technology \\ Taipei, Taiwan \\ sfsu@mail.ntust.edu.tw, maximchen.chen@gmail.com
}

\begin{abstract}
The paper proposes a novel fuzzy system structure to enhance the performance of fuzzy neural network systems. The structure of enhanced fuzzy system (EFS) is to decompose each fuzzy variable into fuzzy subsystems called component fuzzy systems to act as type 2 fuzzy, and each component fuzzy system is based on one traditional fuzzy set with one pair of symmetry fuzzy sets. In addition, in order to illustrate the performance of EFS, the paper utilizes the common back propagation learning algorithm for neural networks in the identification of dynamic systems. From simulation results, it is evident that the proposed EFS have much faster convergent speed in terms of epochs in the tracking model and better testing error than those of using other identification methods.
\end{abstract}

Keywords: Enhanced fuzzy systems; type-2 fuzzy set; back propagation; neural network systems;

\section{Introduction}

In recent years, increasing attention has been given to fuzzy systems in the field of system identification and control because of its simplicity and flexibility. The stability analysis and design of fuzzy control systems had been proposed by using a Lyapunov function candidate in [1]. This kind of fuzzy control methodology is employed in many control design applications as adaptive fuzzy control [2], sliding fuzzy control [3], fuzzy backstepping control [4], etc. Besides, several self-structuring algorithms have been proposed to obtain a possible and appropriate antecedent-part. Since the antecedent-part design is of free structure, the self-structuring algorithm is difficult to mathematically explain. For this reason, the derivative free based optimization approaches are often used in the selfstructuring learning algorithm design. However, the IFThen rules in systems with a high degree of uncertainty are hard to define properly. When the rules cannot clearly be defined, the capability of function approximation may not be good enough and the learning results may not be efficient. To overcome this problem, Zadeh introduced the type- 2 fuzzy logic systems in 1975 [5].
It can be found that because the type-2 fuzzy system (FS) can provide more design degrees of freedom and parameters, it is usually proposed to handle higher degree of uncertainties. In general, the membership function of the type-2 FS is of three dimensions that include upper membership function (MF), lower MF, and a footprint of uncertainty (FOU). Upper and lower MFs are similar to the type- 1 MFs, and FOU can provide an additional degree of freedom that makes the type-2 FS possible to handle uncertainties directly. However, there is no any idea on how to make the best choice on these secondary (Upper or lower) MFs. Although most researchers indicated that the performance of type-2 FS could be better than that of the type- 1 FS, the computational cost associated with type-2 FS is high. Thus, a simplified type-2 FS [6-10] called the interval type- 2 FS was proposed. The performance of interval type-2 FS can achieve similar results with traditional type-2 FS, but the computational cost is greatly reduced. The only difference is that the output processing includes an extra type-reducer, which is employed to convert type- 2 FS into a type- 1 FS. Then, it can be processed by using the defuzzifier to obtain a crisp value output. Although type-reducer is an easy way to resolve a complicated computation problem, the design degrees of freedom are also restricted. This paper proposes a novel fuzzy structure called enhanced fuzzy system (EFS). This structure is originally proposed to replace a traditional fuzzy system and is employed for control [11]. In the structure, the fuzzy variables are decomposed into several subsets and each subset includes one traditional fuzzy set with one pair of symmetry fuzzy sets. To combine all variables forms the so-called component fuzzy systems. The structure of EFS is proposed to facilitate minimum distribution learning effects among component fuzzy systems so that the learning capability can be very efficient. In this paper, we also use this idea to consider type- 2 FS. It can be found that the idea is simple and straightforward and without introducing any extra operation it can still achieve high degree of uncertainty.

This paper is divided into five main sections. Section 1 provides some background information about the EFS. Section 2 outlines the method of the EFS and interval type-2 fuzzy systems. The structure of enhanced fuzzy systems (EFSs) is included in Section 3. Section 4 presents an example to verify the effectiveness of the 
proposed method. Finally, conclusive remarks are given in Section 5.

\section{Interval Type-2 fuzzy system and Enhanced fuzzy system}

In this section, a brief overview and discussion of type2 fuzzy system is presented. The interval type-2 FS is of three dimensions that includes upper membership function, lower membership function, and a footprint of uncertainty (FOU). The interval type-2 membership function is shown in Fig. 1. The structure of the interval type-2 FS is shown in Fig. 2. In the architecture of enhanced fuzzy system, the fuzzy variable is decomposed into $N_{s}$ layers, and each layer includes one type-1 fuzzy sets with one pair symmetrical fuzzy sets, as shown Fig. 3. $A_{2}^{(1)}$ is presented as an original fuzzy set. $A_{1 \_1}^{(1)}$ and $A_{3 \_1}^{(1)}$ are the complement fuzzy sets. Then, all possible combinations of those layers can form $N_{c}=\left(N_{s}\right)^{n}$ fuzzy sub-systems. $n$ is the number of input fuzzy variables. It can be seen that each fuzzy subsystem is of parallel processing can is independence to others in the learning process.

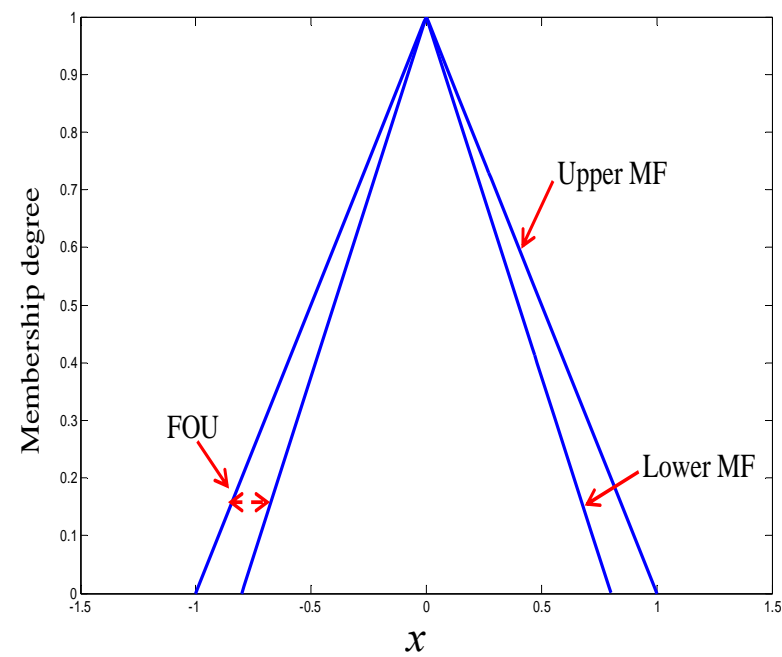

Fig. 1: Illustration of an interval type-2 membership function

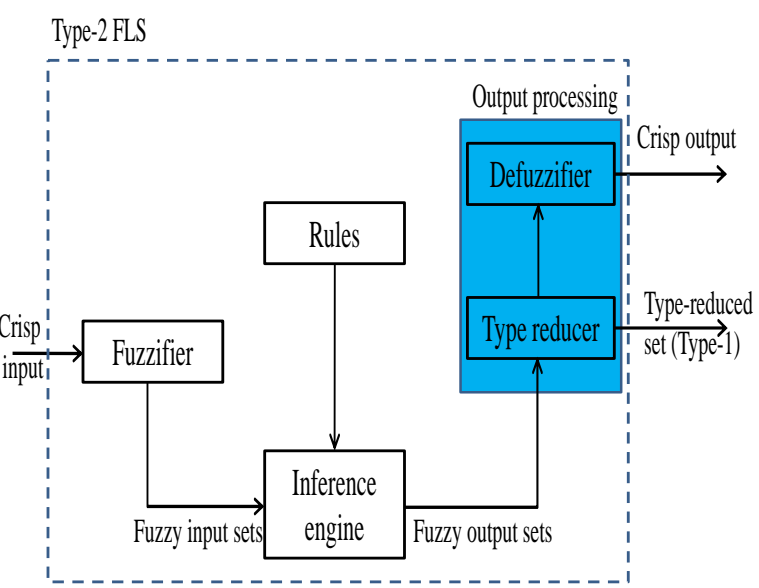

Fig. 2: Structure of the interval type-2 fuzzy systems

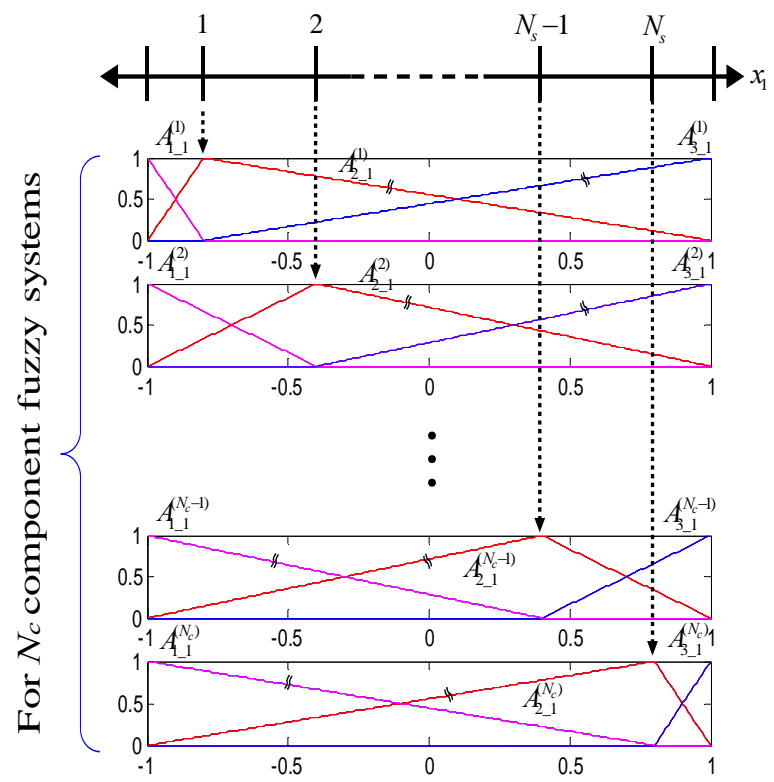

Fig. 3: Antecedent-part design of EFS

Now, the $l$-th fuzzy IF-THEN rule of the $s$-th subsystem is expressed as

$$
\begin{gathered}
R_{(s), l} \text { :If } x_{1} \text { is } A_{1}^{(s), l} \text { and } x_{2} \text { is } A_{2}^{(s), l} \text { and } \\
\quad \ldots x_{n} \text { is } A_{n}^{(s), l} \text { Then } y_{p} \text { is } \theta_{(s), l}
\end{gathered}
$$

where $\mathbf{x}=\left[x_{1}, x_{2}, \cdots, x_{n}\right]^{T} \in R^{n}$ is the input variable vector and $y_{p}$ is the output variable. $l$ is fuzzy number. $A_{n}^{(s), l}$ and $\theta_{(s), l}$ are the corresponding input and output fuzzy sets. $\left.A_{j}^{(s), l}\right|_{j=1, \cdots, n}$ are those $\left.A_{k_{-j} j}^{(s)}\right|_{j=1, \cdots, n ; k=1, \ldots, 3}$ in Fig. 2. Note that in this study, those $\theta_{(s), l}$ 's are fuzzy singletons and are parameters to be tuned in the learning process. According to the center-average defuzzification and the product inference, the output $y_{p(s)}$ of the fuzzy sub-system is

$$
y_{p(s)}=\frac{\sum_{l=1}^{N_{r}} \theta_{(s), l}\left[\prod_{j=1}^{n} \mu_{A_{j}^{(s), l}}\left(x_{j}\right)\right]}{\sum_{l=1}^{N_{r}}\left[\prod_{j=1}^{n} \mu_{A_{j}^{(s), l}}\left(x_{j}\right)\right]}=\boldsymbol{\theta}_{(s)}^{T} \boldsymbol{\omega}_{(s)}
$$

where $\mu_{A_{j}^{(s), l}}\left(x_{j}\right)$ is the membership function values of the fuzzy sets $A_{j}^{(s), l}$ and $N_{r}=(3)^{n}$ is the IF-THEN rules in one fuzzy sub-system. $\boldsymbol{\theta}_{(s)}^{T}=\left[\theta_{(s), 1}, \theta_{(s), 2}, \cdots, \theta_{(s), N_{r}}\right]$ is the adjustable parameter vector. $\boldsymbol{\omega}_{(s)}=\left[\omega_{(s), 1}, \omega_{(s), 2}, \cdots, \omega_{(s), N_{r}}\right]^{T}$ is a fuzzy basis vector, where $\omega_{(s), l}$ is expressed as

$$
\left.\omega_{(s), l}\right|_{l=1,2, \cdots, N_{r}}=\frac{\prod_{j=1}^{n} \mu_{A_{j}^{(d), l}}\left(x_{j}\right)}{\sum_{l=1}^{N_{r}} \prod_{j=1}^{n} \mu_{A_{j}^{(d), l}}\left(x_{j}\right)} .
$$

The average of the outputs of all fuzzy sub-systems is calculated as the output of the enhanced FS hence the output $y_{p f}$ of all fuzzy sub-systems can be defined as 


$$
y_{p f}=\left(\sum_{d=1}^{N_{c}} y_{f(d)}\left(\mathbf{x} \mid \boldsymbol{\theta}_{(d)}\right)\right) / N_{c},
$$

where $N_{c}=\left(N_{d}\right)^{n}$ is the number of fuzzy subsystems. The general term of (4) can be represented as

$$
y_{p f}=\sum_{d=1}^{N_{c}} y_{f(d)} \alpha_{c},
$$

where $\sum_{d=1}^{N_{c}} \alpha_{c}=1$. However, there is no way of evaluating which fuzzy sub-systems may be more important than the others. Thus, in this study, those fuzzy subsystems are equally treated and then will have the same weights; that is, $\alpha_{c}=1 / N_{c}$ as in (5).

From the above structure, it can be observed that the proposed structure is to use a fuzzy system (an original fuzzy and its complement fuzzy sets) to model the type2 fuzzy. Thus, the modeling of a type- 2 fuzzy becomes the modeling of a component fuzzy system. It also can be found that the reasoning process of EFS is the same as the original fuzzy system. Thus, it can be said that the proposed system can model type-2 fuzzy without extra operations in the type reduction. From later simulation, it can also be found that the proposed system can indeed provide a better modeling performance than the original type-2 fuzzy system does.

\section{Enhanced Fuzzy Neural Network System}

Fuzzy neural network systems [12-14] have been wildly used in the identification of unknown models. The aim of the study is to propose a new structure as the enhanced fuzzy system. The detailed algorithm of enhanced fuzzy neural network system (EFNNS) is introduced in this section.

\subsection{Layered Operation of the Enhanced Fuzzy Neural Network System}

In this part, the algorithm of the enhanced fuzzy neural network systems is divided into four layers including the input layer, the linguistic term layer, the rule layer, and the output layer. We are going to describe respectively in the following.

Layer 1 (Input layer): For the $j$ th node of the layer one, each node represents an input linguistic variable $x_{j}$,

$$
\begin{aligned}
& x_{j}=x_{1}, x_{2}, \ldots, x_{n} . \\
& \qquad O_{(s), j}^{1}=x_{j} .
\end{aligned}
$$

Layer 2 (Linguistic term layer): A commonly-used triangular membership function is used for each node in this layer. Those layers perform the fuzzification process. For the jth fuzzy sets $\left.A_{j}^{(s), l}\right|_{j=1, \ldots, n}$ in the input variable $x_{j}$, the membership function is expressed as

$$
\mu_{A_{j}^{(d,) l}}\left(O_{(s), j}^{1}\right)=O_{(d), j}^{2} .
$$

Layer 3 (Rule layer): The nodes in this layer are called rule nodes and perform the product operation. Thus, the $j$ th rule node describes as

$$
O_{(s), j}^{3}=\prod_{j=1}^{n} O_{(s), j}^{2}
$$

Layer 4 (Output layer): In this layer, each node corresponds to one output node. This layer performs the defuzzification process. The node operations are

$$
O_{p f}^{4}=\frac{\sum_{s=1}^{N_{c}} \sum_{l=1}^{N_{r}} \theta_{(d), l} O_{(s), j}^{3}}{N_{c} \sum_{l=1}^{N_{r}} O_{(s), j}^{3}},
$$

where $\theta_{(s), l}$ is the adjustable parameter vector and $l$ is the rule number.

\subsection{Learning Algorithm}

Consider the single-output case. The task is to minimize the cost function

$$
E(k)=\frac{1}{2} \sum_{k=1}^{P}\left[d(k)-y_{p f}(k)\right]^{2}=\frac{1}{2}[e(k)]^{2},
$$

where $d(k)$ is the desired output for pattern $k$ and $y_{p f}(k)=O_{p f}^{4}$ is the current output of the enhanced fuzzy system for pattern $k . P$ is the training pattern number. By using back propagation learning algorithm, $\boldsymbol{\theta}(t)$ can be adjusted in the training process at time $t$. Thus, the weight update law of back propagation algorithm can be writing as

$$
\boldsymbol{\theta}(t+1)=\boldsymbol{\theta}(t)+\Delta \boldsymbol{\theta}(t)=\boldsymbol{\theta}(t)+\eta\left(-\frac{\partial E(t)}{\partial \theta_{l}(t)}\right),
$$

where $\eta$ is the learning rate and $\boldsymbol{\theta}$ is tuning parameters of the EFS. Then, the gradient of $E(t)$ is

$$
\begin{aligned}
& \frac{\partial E(t)}{\partial \theta_{l}(t)}=\frac{\partial E(t)}{\partial y_{p f}(t)} \frac{\partial y_{p f}(t)}{\partial \theta_{l}(t)} \\
& =-e(t) \frac{\partial y_{p f}(t)}{\partial \theta_{l}(t)}=-e(t) \omega(t)
\end{aligned}
$$

where $e(t)=d(t)-y_{p f}(t)$.

It should be noted that the idea of using EFS is to have fixed an antecedent part. Thus, the back propagation learning algorithm is applied to the consequence part only. Thus, with (5) and (10), the weight update law can be expressed as

$$
\begin{aligned}
& \boldsymbol{\theta}(t+1)=\boldsymbol{\theta}_{l}(t)-\eta \frac{\partial E(t)}{\partial \boldsymbol{\theta}(t)} \\
& =\boldsymbol{\theta}(t)-\eta \frac{\partial E(t)}{\partial y_{p f}(t)} \frac{\partial y_{p f}(t)}{\partial \theta_{l}(t)}=\boldsymbol{\theta}(t)-\eta e(t) \boldsymbol{\omega}(t)
\end{aligned}
$$

\section{Simulation results}

Considering the use of the EFS for identification of dynamic systems. The following nonlinear plant is considered

$$
y_{p}(k+1)=-P y_{P}^{2}(k)+Q y_{P}(k-1) .
$$


Eq.(14) produces a chaotic strange attractor with $P=1.4$ and $Q=1.4$. In this example, the inputs are $y_{p}(t)$ and $y_{p}(t-1)$ and the output is $y_{p}(t+1)$. The initial states is $\left[y_{p}(0), y_{p}(1)\right]=[0.4,0.4]$. There are 2000 patterns in which 1000 patterns are used for training and the remaining 1000 patterns are used for testing. Fig. 4 shows training signals of the dynamic system. The reference signals are shown with blue stars and the training results of using EFS are shown with red stars. The termination condition is the error variation less than 1e-005. Fig. 5 shows the testing data of the dynamic system, where the reference signals are shown with blue stars and EFS results are shown with red stars. Table 1 summarizes the total number of parameters used, the number of training epochs, the training RMSE, and the testing RMSE. The performance of EFS is compared to those using other identification systems, including a FNNS and a IT2FNNS. The EFS can quickly make the training error to approach the termination conditions in 184 epochs. Fig. 6 shows the training RMSE of EFS, FNNS, and IT2FNNS. From the simulation results, it is evident that the EFS has better convergence ability and much faster convergent speed in terms of epochs than that of other systems.

\begin{tabular}{|l|c|c|c|}
\hline Models & FNN & IT2FNN & EFS \\
\hline Fuzzy rules & 25 & 50 & 225 \\
\hline Epoch & 438 & 379 & 184 \\
\hline Training RMSE & $3.11 \mathrm{e}-002$ & $3.0205 \mathrm{e}-002$ & $1.72 \mathrm{e}-002$ \\
\hline Testing RMSE & $3.23 \mathrm{e}-002$ & $3.4305 \mathrm{e}-002$ & $2.61 \mathrm{e}-002$ \\
\hline
\end{tabular}

Table 1. Performance of EFS, FNN, and IT2FNN

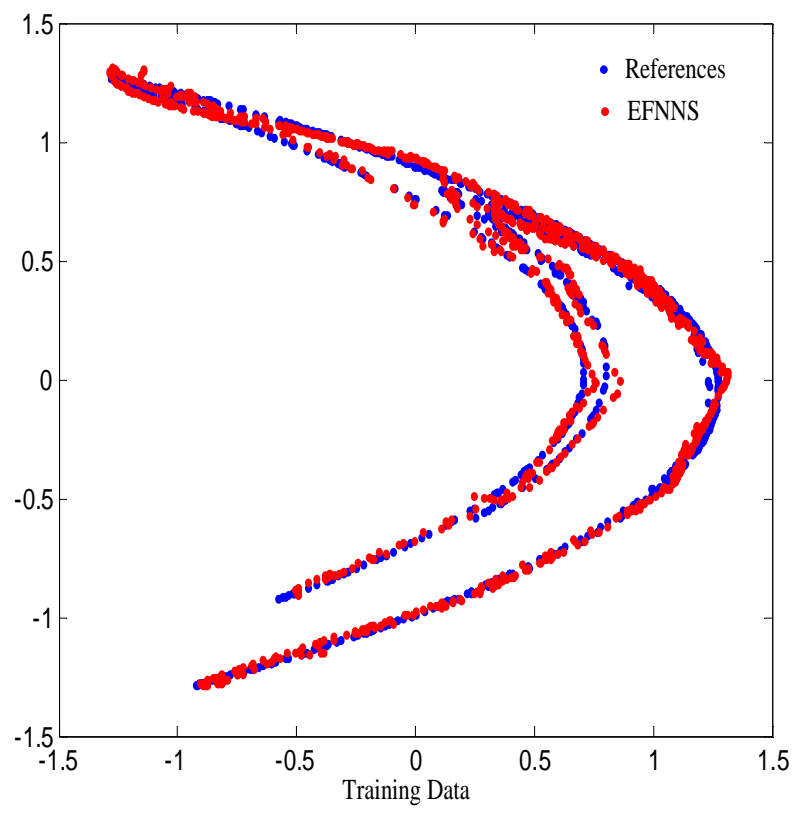

Fig. 4: Results of the training

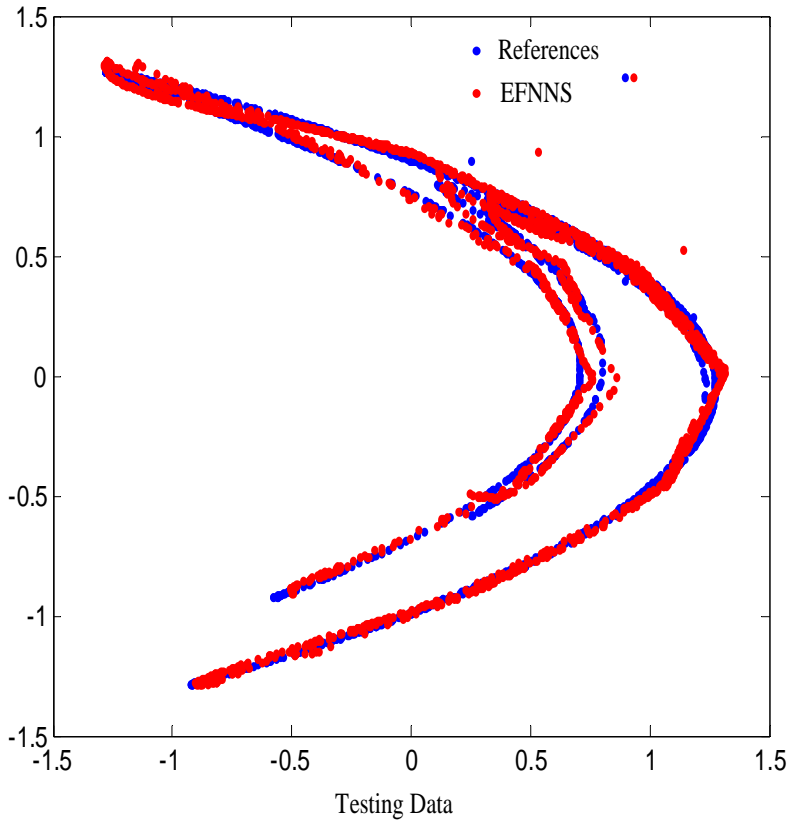

Fig. 5: Results of the testing

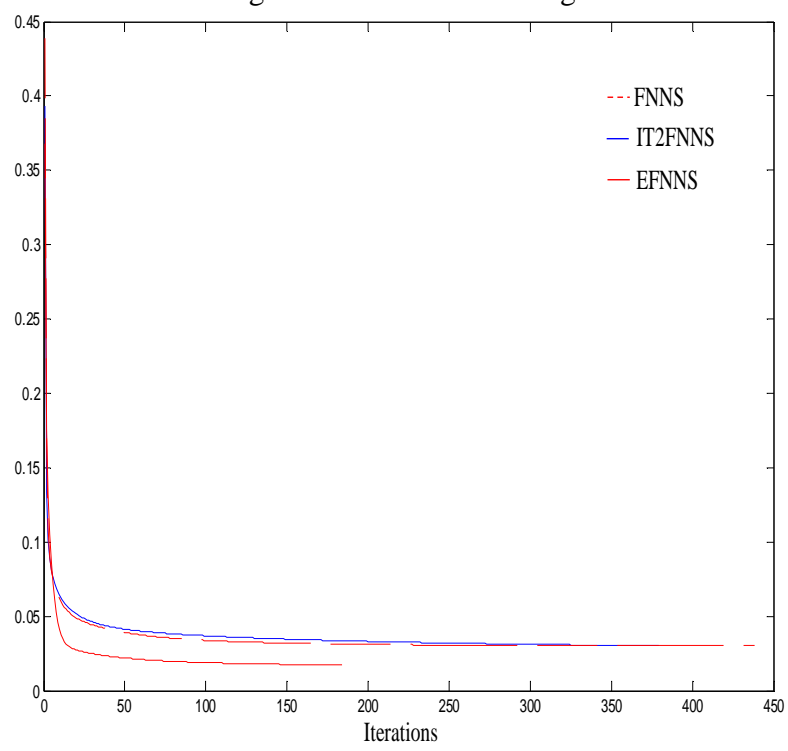

Fig. 6: RMSE histories of all identification methods

\section{Conclusions}

Instead of using traditional fuzzy system structure, a novel fuzzy system structure is proposed in the paper. The structure is combined neural network learning algorithm with enhanced fuzzy systems (EFS). The EFS is composed of multiple component fuzzy systems and each component fuzzy system is based on one traditional fuzzy set with one pair of symmetry fuzzy sets. It can also be found that the function approximation capability and learning efficiency of the EFS are much better than that of the traditional fuzzy systems when employed in enhanced fuzzy systems. In our simulation results, much faster convergent speed in terms of epochs and a good testing error can be obtained by using the proposed structure. 


\section{Acknowledgment}

This work was supported by the National Science Council and Ministry of Science and Technology, Taiwan, under Grant NSC 101-2221-E-011-077-MY3 and MOST 103-2811-E-011-020.

\section{References}

[1] G. Feng, S. G. Cao, N. W. Rees, and C. K. Chak, "Design of fuzzy control systems with guaranteed stability,” Fuzzy Sets Syst., vol. 85, no. 1, pp. 1-10, Jan. 1997.

[2] L. X. Wang, "Stable adaptive fuzzy control of nonlinear systems,” IEEE Trans. Fuzzy Syst., vol. 1, no. 2, pp. 146-155, May 1993.

[3] Ha, Q. H. Nguyen, D. C. Rye, and H. F. DurrantWhyte, "Fuzzy Sliding-Mode Controllers with Applications," IEEE Trans. on Industrial Electronics, vol. 48.no.1, pp. 38-46, Feb. 2001.

[4] S. Tong, Y. Li, Y. Li, and Y. Liu, “Observer-Based Adaptive Fuzzy Backstepping Control for a Class of Stochastic Nonlinear Strict-Feedback Systems," IEEE Trans. on System, Man, and Cybernetics-Part B: Cybernetics, vol. 41, no. 6, pp. 1693-1704, Dec. 2011.

[5] L. A. Zadeh, "The Concept of a Linguistic Variable and Its Application to Approximate Reasoning-1," Information Sciences, vol. 8, pp. 199-249, 1975.

[6] H. Wu and J. M. Mendel, "Uncertainty bounds and their use in the design of interval type-2 FSs," IEEE Trans. Fuzzy Syst., vol. 10, no. 5, pp. 622639, Oct. 2002.
[7]H. Ying, “General interval type-2 Mamdani fuzzy systems are universal approximators," in Proc. of the IEEE Int'l Conf. Information Processing Society, pp. 1-6, 2008.

[8]H. Zhou, H. Ying, and J. Duan, "Adaptive control using interval type-2 fuzzy logic," in Proc. of the IEEE Int'l Conf. Fuzzy Systems, pp. 836-841, 2009.

[9] Y.-Y. Lin, J.-Y. Chang, and C.-T. Lin, "A TSKType-Based Self-Evolving Compensatory Interval Type-2 Fuzzy Neural Network (TSCIT2FNN) and Its Applications," IEEE Trans. on Industrial Electronics, vol. 61, no. 1, Jan. 2014.

[10] O. Castillo and P. Melin, "A review on interval type-2 fuzzy logic applications in intelligent control," Information Sciences, vol. 279, pp. 615-631, 2014.

[11] Y.-C. Hsueh, S.-F. Su, and M.-C. Chen, "Decomposed Fuzzy Systems and Their Application in Direct Adaptive Fuzzy Control," IEEE Trans. on Cybernetics, vol. 44, no. 10, pp. 1772-1783, Jan 2014.

[12] S. Horikawa, T. Furuhashi, and Y. Uchikawa, "On fuzzy modeling using fuzzy neural networks with the backpropagation algorithm," IEEE Trans. Neural Networks, vol. 3, pp. 801-806, Sept. 1992.

[13] K. Tanaka, M. Sano, and H. Watanabe, "Modeling and control of carbon monoxide concentration using a neuro-fuzzy technique," Fuzzy Sets and Systems, vol. 3, pp. 271-279, Aug. 1995.

[14] Y. Lin and G. A. Cunningham, "A new approach to fuzzy-neural system modeling," IEEE Trans. Fuzzy Systems, vol. 3, pp. 190-197, May 1995. 